\title{
Evaluation of Microalbuminuria and Glycosylated Hemoglobin in the Assessment of Diabetes Control in Children with Type 1 Diabetes Mellitus Hospitalized with Diabetic Ketoacidosis
}

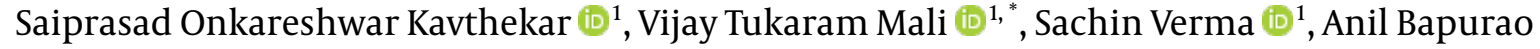 \\ Kurane (iD ${ }^{1}$, Nivedita Balasaheb Patil (iD) ${ }^{1}$ and Suhas Panditrao Kulkarni (iD ${ }^{1}$ \\ ${ }^{1}$ Department of Pediatrics, D.Y. Patil Medical College and Hospital, Kadamwadi, Kolhapur, Maharashtra, India \\ "Corresponding author: Department of Pediatrics, D.Y. Patil Medical College and Hospital, Kadamwadi, Kolhapur (416003), Maharashtra, India. Email: dr.vijaymali@gmail.com
} Received 2020 October 02; Revised 2021 February 21; Accepted 2021 April 10.

\begin{abstract}
Background: Microalbuminuria is thought to be an early predictor of impending diabetic nephropathy, while glycosylated hemoglobin (HbA1c) is a biochemical marker of long-term glycemic control in children with type 1 diabetes mellitus (T1DM).

Objectives: The study aimed to evaluate the prevalence of microalbuminuria and its association with HbA1c on admission and duration of diabetes. Also, changes in HbA1c level on admission and three months after admission were studied to assess diabetes control in children with T1DM.

Methods: This prospective study was conducted among 38 children ( $<18$ years) diagnosed with TIDM presenting with clinical signs, symptoms, and biochemical parameters of DKA. The presence of microalbuminuria, HbA1c level, and the number of past episodes of DKA were recorded. HbA1c level was again estimated after three months. The resultant data was tabulated and analyzed statistically $(\mathrm{P}<0.05)$.

Results: Microalbuminuria and poor HbA1c control were observed in $18 \%$ and $60 \%$ of the sample population, respectively. A significant association was found between HbA1c $>9 \%(\mathrm{P}=0.032)$ and the duration of diabetes $>4$ years $(\mathrm{P}=0.032)$ and microalbuminuria. Significant improvement in glycemic control was noted from the time of admission to three months after admission $(9.76 \pm 2.77 \mathrm{vs}$. $7.75 \pm 1.28 ; \mathrm{P}=0.00012)$. A significant difference was observed between past DKA episodes according to HbA1c control $(\mathrm{P}<0.001)$. Conclusions: Microalbuminuria assessment is needed in T1DM children, especially those with HbA1c $>9 \%$ and duration of diabetes $>$ four years, to evaluate diabetic nephropathy. Good glycemic control can be achieved with effective insulin therapy accompanied by appropriate counseling and regular follow-up.
\end{abstract}

Keywords: Diabetic Ketoacidosis, Glycosylated Hemoglobin, Microalbuminuria

\section{Background}

Type 1 diabetes mellitus (T1DM) is a chronic autoimmune disease characterized by elevated blood glucose levels resulting from insulin deficiency (1). The prevalence of T1DM in the Indian subcontinent is increasing, and it ranges from 3.7 to 10.2 per 100,000 population, with a higher prevalence in urban areas and in males (2).

Acute complications of diabetes such as hyperglycemia and diabetic ketoacidosis (DKA) are the prime causes of increased morbidity and mortality. However, microvascular and macrovascular changes cause significant mortality over time (3). Literature reveals that patients with DKA and those with nephropathy have higher mortality rates (4). Diabetic nephropathy usually develops approximately ten years post diagnosis of T1DM (5). Microalbuminuria is an early predictor of diabetic nephropathy and is associated with higher levels of glycosylated hemoglobin (HbA1c) (5). HbA1c is a biochemical marker that is used routinely in diabetic patients to monitor long-term glycemic control and assess the risk of developing complications.

Urine microalbumin and HbA1c play an important complementary role, and their availability for clinical use together with improvement in therapies would help improve metabolic control in diabetic children. However, there is a paucity of research and guidelines concerning the association of microalbuminuria and HbA1c in T1DM children, especially in Indian subcontinent. 


\section{Objectives}

This study aimed to evaluate the prevalence of microalbuminuria and its association with HbA1c on admission and duration of diabetes in DKA children. Also, changes in the level of HbA1c on admission and three months after admission were studied to assess diabetic control. Additionally, the study evaluated the correlation between HbA1c level and past episodes of DKA.

\section{Methods}

The prospective study was conducted at the Department of Pediatrics at a tertiary care hospital in Kolhapur, India from 1st January 2018 to 31st March 2020, after obtaining ethical clearance from the institutional Ethics committee. Thirty-eight children less than 18 years of age with debut or established T1DM admitted to the Pediatric Intensive Care Unit were recruited into the study after obtaining written informed consent from their parents/guardians.

Detailed history including the duration of diabetes in established T1DM children along with clinical examination and investigations such as blood glucose, arterial blood gases, serum beta-hydroxy butyrate, serum electrolytes, urine sugar, and urine ketone bodies were recorded. Diabetic ketoacidosis was defined as blood glucose $>200$ $\mathrm{mg} / \mathrm{dL}$ with venous $\mathrm{pH}<7.3$ and bicarbonate $<15 \mathrm{mmol} / \mathrm{L}$ and ketonemia (blood $\beta$-hydroxybutyrate concentration $\geq 3 \mathrm{mmol} / \mathrm{L}$ ) or moderate or severe ketonuria (6).

The number of past episodes of DKA in each of the subjects was recorded. Microalbuminuria is defined as albumin to creatinine ratio (ACR) between 30 and $299 \mathrm{mg} / \mathrm{g}$ (7) However, it was assessed on the first morning using $5 \mathrm{~mL}$ of spot urine sample, collected in a clean plastic container. The ACR ratio was calculated in $\mathrm{mg}$ albumin/gm of creatinine. Urinary albumin was estimated by the immuneturbidimetric end-point method by using Bio Lab diagnostic reagents (8) Urinary creatinine was estimated by Jaffe's method using Transasia Bio-Medicals reagents. (9) Microalbuminuria was confirmed by repeating the ACR ratio test after 48 hours using $5 \mathrm{~mL}$ of fresh morning's first urine spot sample.

While testing for ACR, the patient was well-hydrated and afebrile to avoid false positivity for microalbuminuria. HbA1c was estimated by the high-pressure liquid chromatography method (Bio-Rad D10 model) by collecting $2 \mathrm{~mL}$ blood sample in an ethylenediamine tetra-acetic acid container at the time of admission and three months afterward during follow-up (10). It was standardized as per the National Glycohemoglobin Standardization Program (NGSP) (11). The American diabetic association guidelines suggest that target HbA1c level should be $<7.5 \%$ in T1DM children less than 18 years (12). The values of HbA1c were categorized for the purpose of this study as good control (6 - 7.5\%), fair control (7.51 - 9\%), and poor control (> 9\%). Patients diagnosed with DKA were managed as per the International Society of Pediatric and Adolescent Diabetes (ISPAD) clinical practice consensus guidelines protocol 2018 (13). After successful management of acute DKA episode, at the time of discharge, all patients were advised to undergo treatment with basal bolus insulin therapy with appropriate counseling and regular follow-up every 15 days.

\subsection{Statistical Analysis}

Data were analyzed using R Studio V 1.2.5001 software. Continuous variables are expressed in mean \pm standard deviation (mean $\pm S D$ ), while categorical variables are expressed as percentage and frequency. Wilcoxon-sign-rank test was used to examine differences between means. Proportion test was used to find the proportion difference. A P-value of less than 0.05 was considered statistically significant.

\section{Results}

The mean age of the children was $7.4 \pm 3.7$ years (range: 5 months to 13 years), and $53 \%$ of the patients were female. Of the total $(n=38)$ hospitalized children, 21.1\% $(n=8)$ and $78.9 \%(n=30)$ had debut and established T1DM, respectively. On admission, $60 \%(n=23)$ of the patients had poor HbA1c control. Microalbuminuria was found in $18 \%$ ( $n=$ 7) of the children, and all these children had established T1DM with the mean duration of diabetes $4.28 \pm 1.25$ years. The youngest child with microalbuminuria was 8 years old, while the oldest was 13 years old. In the children with microalbuminuria, the average level of HbA1c was > 9\%. A significant association was found between urine microalbuminuria and HbA1c $>9 \%(P=0.032)$ and duration of diabetes $>4$ years $(\mathrm{P}=0.032$; Table 1$)$.

Three months after treatment, the status of HbA1c was improved from poor control to good and fair control (Table 2). Similarly, a significant difference was found between the mean HbA1c value at the time of admission and the same value three months after treatment ( $9.76 \pm 2.77$ vs. $7.75 \pm 1.28 ; \mathrm{P}=0.00012$ ).

The mean number of past DKA episodes was $2.85 \pm$ 1.74. The mean number of past DKA episodes in "good HbA1c control" patients was less (1.3 per person) than in "fair HbA1c control" (2.8 per person) and "poor HbA1c control" patients (2.4 per person). A significant proportion difference was observed between the number of past DKA episodes $\left(\mathrm{P}=1.363 \mathrm{e}^{-13}\right)$ according to HbA1c control. The distribution of past DKA episodes according to HbA1c is shown in Table 3. 


\begin{tabular}{|c|c|c|c|}
\hline & \multicolumn{3}{|c|}{ Association between Microalbuminuria and HbA1c } \\
\hline \multirow{5}{*}{$\begin{array}{l}\text { Microalbuminuria } \\
\text { Present }(\mathbf{n}=\mathbf{0 7})\end{array}$} & $\mathbf{H b A 1 c}(\geq \mathbf{9} \%)$ & $\operatorname{HbA1c}(<9 \%)$ & P-Value \\
\hline & $6(86)$ & $1(14)$ & 0.032 \\
\hline & \multicolumn{3}{|c|}{ Association between Microalbuminuria and Duration of Diabetes } \\
\hline & Duration of Diabetics $(\geq \mathbf{4} y)$ & Duration of Diabetics $(<\mathbf{4}$ y) & P-Value \\
\hline & $6(86)$ & $1(14)$ & 0.032 \\
\hline
\end{tabular}

${ }^{\mathrm{a}}$ HbA1c: Glycosylated Hemoglobin

\begin{tabular}{lccc}
\hline Table 2. Distribution of Patients Before and After Treatment According to HbA1c ${ }^{\mathrm{a}}$ Category & & \\
\hline HbA1c & Number of Patients at the Time of Admission (\%) & Number of Patients at 3 Months After Treatment (\%) & P-Value \\
\hline Good control & $09(24)$ & 0.055 \\
Fair control & $06(16)$ & 0.012 \\
Poor control & $23(60)$ & $0.001(47)$ \\
\hline
\end{tabular}

${ }^{a}$ HbA1c: Glycosylated hemoglobin

\begin{tabular}{lccc}
\hline Table 3. Past Diabetic Ketoacidosis Episodes According to HbA1 ${ }^{\mathrm{a}}$ & & \\
\hline HbA1c & Total Number of DKA Episode & Percentage (\%) & $\begin{array}{c}\text { Number of DKA Episodes per } \\
\text { Person }\end{array}$ \\
\hline Good & 12 & 14.29 & 1.3 \\
Fair & 17 & 20.24 & 2.8 \\
Poor & 55 & 65.48 & 2.4 \\
\hline
\end{tabular}

${ }^{\mathrm{a}}$ HbA1c: Glycosylated hemoglobin

\section{Discussion}

Type 1 diabetes mellitus increases the risk of microvascular complications, such as nephropathy, retinopathy, and neuropathy. Increased incidence of T1DM with the burden of microvascular complications can influence the prognosis of young patients. Microalbuminuria is an early clinical indicator of diabetic nephropathy and is a predictor of increased cardiovascular mortality and morbidity (14). This study was undertaken to evaluate the prevalence of microalbuminuria and its association with HbA1c and to assess level of HbA1c in T1DM children with DKA on admission and three months after admission to assess diabetic control. Additionally, the study evaluated the correlation between HbA1c level and the number of past DKA episodes.

In this study, the demographic characteristics such as age and gender were commensurate with the characteristics reported in literature (15). In this study, microalbuminuria was present in $18 \%$ of the subjects, and all of these had poorly controlled glycemic levels. Various studies have found the prevalence of microalbuminuria in T1DM to range from $3.3 \%$ to $29.6 \%$ (16). The occurrence of microalbuminuria is dependent on various factors such as age, gender, duration of diabetes, glycemic control, insulin dose, and lipid profile of the patient. Poorly controlled diabetes has been consistently found to be associated with microalbuminuria in various studies (17-19). Checking for the presence of microalbuminuria is recommended after five years of diabetes in prepubertal children and after two years in post pubertal patients (15). In previous studies, it has been suggested that the presence of persistent microalbuminuria among diabetic patients may require annual assessment for the presence of microalbuminuria (17).

Diabetic ketoacidosis is a common presentation in T1DM patients, and it arises due to the lack of adequate insulin in the body resulting in lipid being used as a source of energy. Insulin deficiency results in increased glucagon levels, which results in the release of free fatty acids from the fatty tissue and amino acids from muscles (20). It is a potentially life-threatening condition with risk factors including children $<2$ years, delayed diagnosis, and patients from lower socioeconomic strata with limited access to healthcare. Diabetic ketoacidosis at the time of diagnosis of diabetes is ominous of long-term poor glycemic control (21). Likewise, in this study, poor and fair diabetes control were associated with higher episodes of DKA when compared with good glycemic control, demonstrat- 
ing that higher HbA1c level is associated with increased morbidity in the form of increased episodes of DKA.

This study also established the need for the evaluation of DKA and microalbuminuria with HbA1c in T1DM patients, as there is a paucity of research in this area. In this study, patient management with insulin therapy with appropriate counseling and regular follow-up resulted in good diabetic control. However, the study has certain limitations as HbA1c level is usually affected by the presence of hemoglobin variants and elevated levels of fetal $\mathrm{Hb}$. In addition, since the study is unicentric, it is not wellgrounded. Thus, a similar multicenter study with a larger sample size and precise assessment of HbA1c would yield more generally applicable results.

\subsection{Conclusions}

Overall, $18 \%$ and $60 \%$ of the patients had microalbuminuria and poor HbA1c control, respectively. Assessment of microalbuminuria is needed in pediatric T1DM patients, especially in those with HbA1c $>9 \%$ and duration of diabetes $>4$ years, to evaluate the risk of diabetic nephropathy. Management with insulin therapy along with appropriate counseling and regular follow-up can significantly contribute to good glycemic control.

\section{Footnotes}

Authors' Contribution: Study concept and design: SOK, VTM, Acquisition of data: SOK, VTM, SV, ABK, NBP, SPK, Analysis and interpretation of data: VTM, Drafting of the manuscript: SOK, VTM, SV, ABK, NBP, SPK, Critical revision of the manuscript for important intellectual content: SOK, VTM, SV, ABK, NBP, SPK, Statistical analysis: SOK, VTM, Administrative, technical, and material support: SOK, VTM, SV, ABK, NBP, SPK, Study supervision: VTM.

Conflict of Interests: The authors declare no conflicts of interest.

Ethical Approval: The study is ethically approved by institutional ethics committee with the registration number DMCK/116/2017.

Funding/Support: The authors declare that there was no funding/support.

Informed Consent: Proper Informed consent was obtained in conducting the study.

\section{References}

1. Kahanovitz L, Sluss PM, Russell SJ. Type 1 diabetes-a clinical perspective. Point of care. 2017;16(1):37-40. doi: 10.1097/poc.0000000000000125.

2. Malik A, Morya RK, Bhadada SK, Rana S. Type 1 diabetes mellitus: Complex interplay of oxidative stress, cytokines, gastrointestinal motility and small intestinal bacterial overgrowth. Eur JClin Invest. 2018;48(11). e13021. doi: 10.1111/eci.13021.
3. de Boer IH, Bakris GL. Diabetic kidney disease: A determinant of cardiovascular risk in type 1 diabetes. Diabetes Care. 2018;41(4):662-3. doi 10.2337/dci17-0053.

4. Baalaaji M, Jayashree M, Nallasamy K, Singhi S, Bansal A. Predictors and outcome of acute kidney injury in children with diabetic ketoacidosis. Indian Pediatr. 2018;55(4):311-4. doi: 10.1007/s13312-018-1274-8.

5. Idowu AA, Ajose AO, Adedeji AT, Adegoke AO, Jimoh KA. Microalbuminuria, other markers of nephropathy and biochemical derangementsin type 2 diabetes mellitus: Relationships and determinants. Ghana Med J. 2017;51(2):56-63. [PubMed: 28955101]. [PubMed Central: PMC5611905].

6. Cashen K, Petersen T. Diabetic ketoacidosis. Pediatr Rev. 2019;40(8):412-20. doi: 10.1542/pir.2018-0231.

7. Muddu M, Mutebi E, Isaac S, Kizito S, Mulindwa F, Mondo Charles K. Microalbuminuria among newly diagnosed diabetic patients at mulago national referral hospital in uganda: A cross sectional study. $J$ obes weight-loss Medic. 2018;4(1). doi: 10.23937/2572-4010.1510021.

8. Molinario R, Pocino K, Daloiso PD, Giannace A, Spirito G, Zuppi C, et al Urinary albumin detection: Comparison of two different methods. $J$ Clin Lab Anal. 2016;30(6):888-91. doi: 10.1002/jcla.21952.

9. Küme T, Sağlam B, Ergon C, Sisman AR. Evaluation and comparison of Abbott Jaffe and enzymatic creatinine methods: Could the old method meet the new requirements? J Clin Lab Anal. 2018;32. e22168. doi: $10.1002 /$ jcla.22168.

10. Chikine RK, Bhumi MA. Method verification procedure of boric acid affinity method against high performance of liquid chromatography for glycated haemoglobin. Int J Clin Biochem Res. 2020;7(3):367-73. doi 10.18231/j.ijcbr.2020.079.

11. Sacks DB, Rohlfing C, Little RR. The National Glycohemoglobin Standardization Program: Over 20 Years of Improving Hemoglobin A1c Measurement. Clin Chem. 2019;65(7):839-48. doi 10.1373/clinchem.2018.296962.

12. American Diabetes Association.12. Children and adolescents. Diabetes Care. 2016;40(Supplement 1):S105-13. doi: 10.2337/dc17-S015.

13. Wolfsdorf JI, Glaser N, Agus M, Fritsch M, Hanas R, Rewers A, et al Ispad clinical practice consensus guidelines 2018: Diabetic ketoacidosis and the hyperglycemic hyperosmolar state. Pediatr Diabetes. 2018;19:155-77. doi: 10.1111/pedi.12701.

14. Kamaleldeen EB, Mohammad HA, Mohamed EF, Askar AG. Microvascular complications in children and adolescents with type 1 diabetes mellitus in Assiut governorate, Egypt. Gaz Egypt Paed Assoc. 2018;66(4):85-90. doi: 10.1016/j.epag.2018.10.003.

15. Al-Eisa AA, Al-Hajri A, Al-Shuaib S, Razzak DMA, Al-Basiri I. Early-onset microalbuminuria in children with type 1 diabetes in Kuwait. Curr Pediatr Res. 2017;21(2):254-9.

16. Aljabri KS, Bokhari SA, Alshareef MA. Modified risk factors for presence of microalbuminuria in saudi adults with type 1 and type $2 \mathrm{di}$ abetes mellitus. J Diabetes Metab Disord Control. 2018;5(4):132-6.

17. Efundem NT, Assob JCN, Feteh VF, Choukem S. Prevalence and associations of microalbuminuria in proteinuria-negative patients with type 2 diabetes in two regional hospitals in Cameroon: a crosssectional study. BMC Research Notes. 2017;10(1):477. doi: 10.1186/s13104017-2804-5

18. Amritanshu K, Kumar A, Anand K, Garg N, Banerjee D. Clinical profile and factors associated with microalbuminuria in type 1 diabetes mellitus in children and adolescents. Int J Res Med Sci. 2015;3(5):1247-51. doi: 10.5455/2320-6012.ijrms20150538.

19. Yarhere IE, Jaja T, Anolue M. Microalbuminuria in type 1 diabetes mellitus children in University of Port Harcourt Teaching Hospital, Nigeria. Pan Afr Med J. 2020;36:161. doi: 10.11604/pamj.2020.36.161.23782.

20. Raghupathy P. Diabetic ketoacidosis in children and adolescents. Indian J Endocrinol Metab. 2015;19(7):55-7. doi: 10.4103/2230-8210.155403.

21. Duca LM, Wang B, Rewers M, Rewers A. Diabetic ketoacidosis at diagnosis of type 1 diabetes predicts poor long-term glycemic control. $\mathrm{Di}$ abetes Care. 2017;40(9):1249-55. doi:10.2337/dc17-0558. 